Cinémas

Revue d'études cinématographiques

Journal of Film Studies

\title{
" Faire le tour de la question »
}

\section{Bernard Perron}

Volume 12, numéro 2, hiver 2002

Cinéma et cognition

URI : https://id.erudit.org/iderudit/024884ar

DOI : https://doi.org/10.7202/024884ar

Aller au sommaire du numéro

\section{Éditeur(s)}

Cinémas

\section{ISSN}

1181-6945 (imprimé)

1705-6500 (numérique)

Découvrir la revue

Citer cet article

Perron, B. (2002). « Faire le tour de la question ». Cinémas, 12(2), 135-157.

https://doi.org/10.7202/024884ar
Résumé de l'article

S'inspirant du cycle perceptif élaboré par Ulric Neisser dans Cognition and Reality (1976), l'auteur transforme ici le modèle habituellement utilisé par l'approche cognitive pour expliquer l'activité perceptivo-cognitive du spectateur, à savoir l'utilisation du mode de perception " de bas en haut " (ou " bottom-up ») et du mode de traitement " de haut en bas » (ou " top-down »). Par la même occasion, l'auteur interroge la manière dont a été théorisée ladite activité et en vient à proposer, pour définir la perception et la compréhension, une expression qu'il considère encore plus appropriée que le « aller au-delà de l'information donnée » de Bruner ou le « recueillir plus d'informations pertinentes » de Neisser. 


\section{«Faire le tour de la question ${ }^{1} »$}

\section{Bernard Perron}

\section{RÉSUMÉ}

S'inspirant du cycle perceptif élaboré par Ulric Neisser dans Cognition and Reality (1976), l'auteur transforme ici le modèle habituellement utilisé par l'approche cognitive pour expliquer l'activité perceptivo-cognitive du spectateur, à savoir l'utilisation du mode de perception "de bas en haut" (ou "bottom-up") et du mode de traitement «de haut en bas» (ou «top-down»). Par la même occasion, l'auteur interroge la manière dont a été théorisée ladite activité et en vient à proposer, pour définir la perception et la compréhension, une expression qu'il considère encore plus appropriée que le «aller au-delà de l'information donnée" de Bruner ou le « recueillir plus d'informations pertinentes " de Neisser.

\section{ABSTRACT}

Being inspired by the perceptual cycle elaborated by Ulric Neisser in Cognition and Reality (1976), the author transforms here the model habitually used by the cognitive approach to explain the perceptive and cognitive activity of the spectator, namely the use of bottom-up and top-down processes. By the same opportunity, the author questions the manner by which the aforementioned activity has been theorized and comes to propose an expression that he considers even more appropriated than the "going beyond the information given" (Bruner), or the "picking up more pertinent information" (Neisser) to define perception and comprehension. 
and a more general schema. It can be regarded as a process of generalizating the object or of particularizing the schema depending on one's theorical inclinations. (Neisser, 1976, p. 65).

Développée à partir des bases jetées par David Bordwell en 1985 dans Narration in Fiction Film et en 1989 dans «A Case for Cognitivism ", la théorie cognitive du cinéma traite le visionnement d'un film comme "a dynamic, perceptual-cognitive activity» (Bordwell, 1985, p. xii). Elle s’intéresse à ce que Julian Hochberg et Virginia Brooks (1996, p. 380-381) ont qualifié de "online perception" et que je nomme pour ma part la spectature-en-progression, à savoir le travail de compréhension qu'effectue le spectateur tout au long d'un film, plus particulièrement d'un film de fiction narratif ${ }^{2}$. Le spectateur se servant des mêmes processus perceptifs et cognitifs pour embrasser et le film et le monde dans lequel il vit, sa compréhension est analysée à partir de notions empruntées à la psychologie cognitive, et plus généralement à la science cognitive. On s'intéresse alors à la perception, à la mémoire, aux schémas de connaissance, aux inférences, au traitement de l'information, à la résolution de problèmes, à la compétence, etc. De façon particulière, on distingue deux types de processus: un processus ascendant, ou "bottom-up ", et un processus descendant, ou «top-down» (voir entre autres Fortin et Rousseau, 1993; Fayol, 1985).

Le mode de perception "bottom-up " est géré ou dirigé par les données perceptives ainsi que par les attributs élémentaires des objets et des événements perçus. Le traitement s'effectue automatiquement et obligatoirement de la partie vers le tout, du particulier au général. Le spectateur essaie de tirer des conclusions à partir de ce qu'il perçoit. Quant au mode de traitement "top-down ", qui tient de la cognition ${ }^{3}$, il est géré ou dirigé par les concepts et les schémas, c'est-à-dire par ces représentations cognitives abstraites qui précisent les propriétés générales d'un type d'objet, d'événement ou de structure, laissant de côté les détails qui ne sont pas pertinents pour caractériser le type. Le traitement s'effectue stratégiquement du tout vers la partie, du général au particulier. Le spectateur construit préalablement une 
représentation de la situation ou du problème en cours et vérifie ensuite si les données y correspondent.

Puisque cette métaphore spatiale empruntée à l'intelligence artificielle ${ }^{4}$ est devenue un modèle pour expliquer l'activité perceptivo-cognitive du spectateur, elle sert de point de départ à ma réflexion. Mais surtout, pour mieux rendre compte de la spectature-en-progression, je souhaite transformer ledit modèle en m'appuyant sur l'ouvrage d'Ulric Neisser, Cognition and Reality (1976), ouvrage dont l'approche cognitive du cinéma a encore très peu tiré profit ${ }^{5}$. Enfin, dans la mesure où, tel que l'a souligné dès le départ Bordwell (1985, p. 30), "[a]ny theory of the spectator's activity must rest upon a general theory of perception and cognition », mes propos déborderont réciproquement le cadre du cinéma.

\section{Le cercle heuristique}

La perception ne se réduit pas à un processus d'identification de stimuli. Au contraire, elle est un phénomène régi par l'anticipation, à savcir un " [...] processus continu d'exploration et de saisie d'informations» (Neisser, 1976, p. 26). De la sorte, affirme Ulric Neisser (1976, p. 43), bien que nous ne puissions pas percevoir, comprendre ou interpréter sans anticipation d'où l'utilisation du mode de traitement "de haut en bas" nous ne devons pas seulement percevoir, comprendre ou interpréter ce que nous anticipons - d'où l'utilisation du mode de perception "de bas en haut". Ce paradoxe nous conduit tout droit au fameux cercle herméneutique tel que l'a décrit à l'origine Schleiermacher (1987, p. 78) (dans son «Herméneutique générale, 1808-1810"): "[...] l'image de la totalité est complétée par la compréhension du détail et [...] le détail est compris de façon d'autant plus complète qu'on a une vue d'ensemble du tout». Je fais référence à l'herméneutique car, comme le déclare Bordwell (1989a, p. 17), "in many, perhaps most, respect, film studies is an hermeneutic discipline. By and large it is the business of interpreting text (mainly, films)». Ce n'est ici pas le lieu pour reprendre le détail du propos de Bordwell dans Making Meaning. Inference and Rhetoric in the Interpretation of Cinema (1989b), mais on retiendra les quatre types de sens qu'il y a défi- 
nis: le sens référentiel (la signification dérive de références réelles ou imaginaires); le sens explicite ou littéral (la signification est clairement donnée par le film); le sens implicite ou symbolique (la signification est indirectement dérivée du film); et le sens symptomatique ou réprimé (la signification est communiquée involontairement). Si, comme l'a bien montré Bordwell, les chercheurs en études cinématographiques se sont intéressés et s'intéressent encore beaucoup au sens implicite et au sens symptomatique, le spectateur, lui, est bien loin de ce que l'on appelle l'herméneutique et que l'on définit entre autres, de manière générale, comme une pratique ou une théorie de l'interprétation qui s'efforce de dévoiler le sens caché des "monuments écrits" (Dilthey, 1947) et les intentions de l'auteur ${ }^{6}$. La spectature-enprogression ne s'efforce cependant pas d'interpréter les films. Elle navigue plutôt sur les eaux du référentiel et de l'explicite et repose aujourd'hui sur "une congénialité perceptuelle importante avec le cinéma" (Thérien, 1992, p. 115). De la sorte, et pour mieux tenir compte de cette compréhension, il mapparaît pertinent de parler d'heuristique. Une heuristique est une méthode d'exploration qui procède par évaluations successives et hypothèses provisoires et qui ne garantit pas une réponse ou une solution claire et précise. Ainsi, le système de communication monodirectionnelle du cinéma n'est pas différent de celui de la communication humaine qu'étudient Dan Sperber et Deirdre Wilson (1989, p. 42 et p. 82-88) dans l'élaboration de leur théorie de la pertinence. Dans les deux cas, il s'agit de communication ostentive-inférentielle. Pour comprendre ce qui est en jeu durant le film, le spectateur doit inférer les visées de la narration à partir des indices qui sont fournis et mis en évidence à cette fin. Tout n'est jamais explicité. La perception en direct du spectateur tient " [...] du mouvement brownien. [Elle] progresse par sauts, retours en arrière, dérives latérales, anticipations et surimpressions, non par caprice ou inconséquence de lecture mais par attention à la trame textuelle" (Gardies, 1993, p. 170). En ce sens, et en relation avec les recherches menées en intelligence artificielle, deux conclusions peuvent être tirées. La première, de Sperber et Wilson (1989, p. 74), c'est que «la plupart des processus cognitifs [impliqués dans la communication] sont si com- 
plexes qu'ils doivent être modélisés par des heuristiques et non par des algorithmes infaillibles". La seconde, d'Annick WeilBarais (1993, p. 50), c'est que «[...] l'intelligence humaine ne réside pas tant dans le calcul mais dans la capacité à construire des représentations adéquates des situations ainsi qu'à les modifier". Sans l'ombre d'un doute, aucun savoir commun, aucun code rigide ne suffit à l'échange et à la compréhension d'une information. Il faut sans cesse tirer des conséquences pour compenser un manque, pour résoudre des incohérences, pour identifier un "script" et une "scène ${ }^{7}$ " ou pour considérer leurs variables. Les inférences à accomplir sont loin d'être toujours bien comprises et loin d'être toutes déductives ou démonstratives, c'est-à-dire sur le mode du «si $\mathrm{P}$, alors $\mathrm{Q}$ ». La plupart du temps, il est nécessaire de formuler ce que Sperber et Wilson nomment des inférences non démonstratives. Celles-ci dépendent et varient selon le contexte. L'interaction entre le spectateur et le film, comme la lecture d'une œuvre de fiction, ressemblent effectivement à un pari ou une gageure - "reading is like a bet", dixit Umberto Eco (1994, p. 112). En conséquence, l'effort intellectuel nécessaire à la compréhension est d'emblée considérable. Mais contrairement à ce que laisse de nouveau entendre Eco tout au long de sa marche — ou analyse posée, devrait-on dire - dans les sentiers de la forêt fictionnelle, ce "spectateur naif de premier niveau» qui progresse n'a pas le mandat d'interpréter le film. S'il est possible d'établir une relation de cette espèce avec la compréhension narrative, c'est au code herméneutique de Barthes qu'il faudrait renvoyer. Ce code, dont le nom n'évite pas les analogies trompeuses, Barthes dans S/Z (1970, p. 24 - voir aussi les sections XI, XXXII, XXXVII et LXXXIX) le définit en ces termes :

\footnotetext{
[...] l'ensemble des unités qui ont pour fonction d'articuler, de diverses manières, une question, sa réponse et les accidents variés qui peuvent ou préparer la question ou retarder la réponse; ou encore: de formuler une énigme et d'amener son déchiffrement.
}

Ainsi, ce dont a besoin le spectateur pour progresser dans cette chaîne des systèmes signifiants, c'est d'un dispositif permettant 
de saisir les tenants et les aboutissants d'une intrigue ou d'une énigme. Un tel dispositif se conçoit aisément depuis le cycle perceptif qu'élabore Ulric Neisser (1976, p. 22-24 et p. 110-117) dans Cognition and Reality pour rendre compte des schémas intégrés aux cartes mentales. Ce cycle, pour poursuivre mon raisonnement et pour mieux l'associer à la spectature-enprogression, je l'appellerai le cercle heuristique (figure $1^{8}$ ).

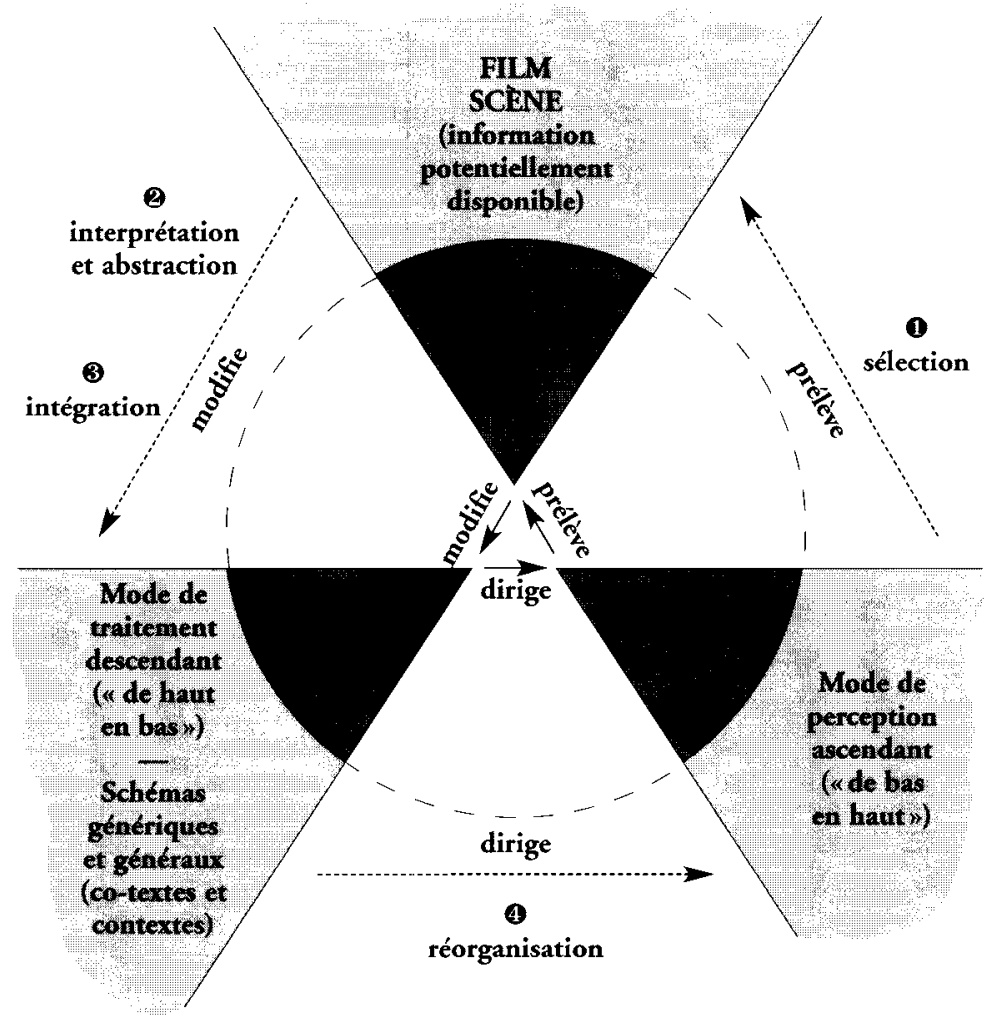

Figure 1

D'entrée de jeu, à la différence de la métaphore spatiale du haut et du bas, le cercle heuristique a l'avantage d'expliciter ce que la psychologie cognitive évoque fréquemment sans y insister, à savoir que le mode de perception ascendant et le mode de traitement descendant ne sont pas des mécanismes indépendants, mais quils fonctionnent en constante interaction et que, 
"[...] selon la nature de la tâche et la connaissance du sujet, l'un des deux peut dominer" (Fayol, 1985, p. 46). Ainsi, si les images et les sons perçus correspondent dans une faible mesure à une expérience passée, le mode ascendant gouverne le cycle perceptif pour prélever des données pertinentes et chercher une structure cognitive appropriée. Par contre, si les hypothèses formulées par l'entremise des données audiovisuelles coïncident avec un schéma de connaissance général (un contexte) ou un schéma narratif générique (un co-texte ${ }^{9}$ ), le mode descendant prend le relais. Celui-ci dirige - il s'agit bien d'une direction et non d'un contrôle puisque l'exploration perceptive demeure indispensable et en continuelle activité - le cercle heuristique et permet de dégager des conclusions non démonstratives. Dans cette foulée, à la différence cette fois de l'énumération diachronique qu'en font les psychologues, ce cercle représente aussi de manière très nette que, au sein de la théorie du schéma, les quatre phases qui régissent la compréhension (et qui jalonnent l'extérieur du cercle) sont toujours cycliques: 1) la sélection: l'information est prélevée en fonction de la disponibilité d'un schéma susceptible de fournir une base d'accueil assimilatrice; 2) l'interprétation et l'abstraction: l'information prélevée est traitée et donc transformée de façon à en conserver une forme plus ou moins abstraite; 3) l'intégration: l'information abstraite est intégrée et comparée avec celles du schéma, schéma qui sera alors confirmé ou modifié; et 4) la réorganisation: le schéma ainsi révisé dirigera la sélection (ou le rejet) de l'information en fonction de sa nouvelle disponibilité (Fayol, 1985, p. 43 et 98; Ellis et Hunt, 1989, p. 184-185).

[C]e cadrage n'introduit pas tant des restrictions quant à ce qui peut faire sens qu'il n'ouvre le champ des possibles. Nous sommes dotés de cette capacité fondamentale d'accepter que les principes organisationnels de notre expérience changent de telle sorte que le sens d'un segment d'activité s'écarte de son modèle tout en restant significatif, capacité qui met en jeu un mécanisme de correction permettant d'éviter que la confusion ne s'installe dans ce que nous percevons de l'intrigue (Goffman [1974] 1991, p. 234). 
$\mathrm{Vu}$ les fluctuations constantes de l'environnement, et celles du temps et de l'espace au cinéma, le mécanisme de correction auquel fait allusion Goffman ne peut qu'être cyclique. Exception faite de Cognition and Reality, et de là l'intérêt et l'influence de la conceptualisation de Neisser, je n'ai trouvé aucun ouvrage de science ou de psychologie cognitive qui introduise une telle figure en boucle. Cette figure expose l'interaction entre le spectateur et le film. Elle permet aussi de mettre intelligiblement en vue que la spectature, engagée dans un tel cycle peceptivocognitif, demeure une activité dynamique en perpétuel mouvement. Enfin, l'intérieur du cercle délimite l'espace de travail du spectateur alors que l'extérieur dénote clairement l'importance de tout son savoir préalable ${ }^{10}$.

Associer la spectature-en-progression à une démarche heuristique, c'est mettre en évidence qu'à l'instar du critique ou du chercheur en études cinématographiques, le spectateur est en quête de connaissance, mais d'une connaissance adaptée à son but premier: progresser dans le récit filmique. Partant, comme toute heuristique, le cercle est employé pour maximiser l'efficacité du traitement, et ce, tant pour la mise en intrigue que pour la mise en film. Bien que je me concentre plutôt, pour ma part, sur la dimension narrative du traitement de l'information, le cercle heuristique s'applique également à la perception et au traitement de l'espace et de la diégèse. Dans ce cas, il est d'ailleurs plus directement lié aux cartes mentales et au cycle perceptif de Neisser. Du reste, comme le note Branigan (1992, p. 38), l'activité perceptivo-cognitive du spectateur se déroule à plus d'un niveau:

Because of the diversity of top-down and bottom-up processes which may be at work at a given moment in a text, perception as a whole is perhaps best thought of as a system which struggles to manage different and often conflicting interpretations of data.

\section{Aller au-delà de l'information donnée}

La phase du cercle heuristique gouvernée par le savoir préalable du spectateur revêt une importance considérable.

How pictures acquire narrative signification cannot be reduced to how surface features and technique are deli- 
neated, or marked by bottom-up perceptual processing, but instead must be analyzed in terms of the top-down cognitive processes which drive us to offer descriptions and apply (macro) propositions to what is seen and heard and read (Branigan, p. 1992, p. 169).

Le film et son intrigue sont manifestement des phénomènes qui relèvent du traitement "de haut en bas" (top-down). Il existe une expression fort répandue en psychologie cognitive pour traduire succinctement ces phénomènes: "going beyond the information given" (Bruner, 1973, p. 218-237 ${ }^{11}$ ). Publique, partagée et créée par l'habituation ou la redondance, la signification est expansive. Pour Bruner, la compréhension ne va pas sans "informations évoquées au-delà du donné", expression constituant un intéressant synonyme d'inférence. Il existe plusieurs variétés d' "informations». Le blanc et le noir éveilleront immédiatement une relation d'opposition. Si je présente à un lecteur français le groupe de lettres « $C^{*} N E^{*} A T^{*} G R A^{*} \mathrm{HE}$ », il reconnaîtra aisément le mot "CINÉMATOGRAPHE»Bruner, lui, utilise " $\mathrm{P}^{*} \mathrm{YC}^{*} \mathrm{OL}{ }^{*} \mathrm{GY}$ " pour "PSYCHOLOGY". Dans son environnement ou dans un domaine particulier, plus une personne possède de connaissances, plus elle sera capable d'«aller au-delà du donné». En 1990, Bruner a étudié avec trois confrères la compréhension narrative (Feldman et al., 1990 , p. 1-78). Leurs expériences ${ }^{12}$ montrent que dans les récits où les agissements des personnages prédominent sur leur profondeur psychologique, les actions reçoivent une organisation causale. Ce sont des mécanismes cognitifs destinés à déchiffrer (to unpack) cette causalité qui sont, dirais-je, mis en branle dans le cercle heuristique. La fiction populaire et le cinéma narratif classique s'associent d'emblée à ce que les psychologues ont nommé le paysage de l'action (landscape of action ${ }^{13}$ ). Les "informations évoquées au-delà du donné" concernent alors les faits futurs et probables. Il serait ainsi tout aussi juste d'affirmer que les informations narratives évoquées vont «au-devant" du donné. Evie (Rachel Weisz), l'héroïne de The Mummy Returns (Stephen Sommers, 2001), a beau être assassinée au deuxième tiers du film, le spectateur est prêt à parier qu'elle sera ressuscitée avant la fin. 
La pierre angulaire de l'expression de Bruner introduite par Bordwell (1989a, p. 18) en études cinématographiques demeure l'adverbe de lieu ou, pour être plus juste, la locution prépositive. Ce fameux "au-delà de", on le retrouve dans plus d'une étude sur la lecture ou la spectature. Même s'il n'est pas associé à l'expression du psychologue cognitiviste, il manifeste d'une façon ou d'une autre que le sens ne se réduit pas à l'espace signifiant délimité par l'information donnée. Par exemple, en appelant le linguiste à aller "au-delà de la phrase», Barthes (1977, p. 11) réclamait l'élargissement de l'étude de la langue au discours. On comprend mieux, dans la perspective de la présente réflexion, qu'il visait la description et l'application macropropositionnelle ${ }^{14}$. S'inspirant de la pensée barthésienne, Branigan (1992, p. 72) en appelle à son tour à un élargissement :

One must expand the notion of a spectator's « knowledge" beyond immediate "seeing" to include various effects produced by the sound track, our memory of previous scenes, anticipated pleasure or anxiety, generic and cultural expectations, and so forth.

C'est qu'on ne peut réduire la connaissance spectatorielle aux informations de surface - Branigan évoque alors «the principle of the "separation of material and structure" "(1992, p. 141142). Au contraire, il faut la rendre plus large, et ce, parallèlement et proportionnellement aux trois niveaux de l'architecture mémorielle: du niveau le plus restreint au plus large, on passera de la mémoire sensorielle à la mémoire de travail, ou mémoire à court terme, et à la mémoire à long terme. Pour exprimer à quel point le spectateur va simultanément au-delà des informations audiovisuelles et au-devant des informations narratives, on ne peut trouver meilleurs exemples cinématographiques que toutes ces occurrences d'allusion, d'économie et d'accélération narratives. Un des paradigmes sémiologiques les plus représentatifs serait la séquence ordinaire de la grande syntagmatique de Christian Metz. Dans ce syntagme, on se contente de "sauter" les moments jugés sans intérêt pour l'intrigue. Tant que l'action est bien définie, comme l'est un trajet entre deux lieux ou un repas pris au restaurant, «ces hiatus diégétiques sont réputés 
insignifiants" (Metz, 1968, p. 133). La séquence ordinaire fait référence à la notion de "script ". On peut omettre des " "scènes" de scripts ${ }^{15}$ " ou laisser des hiatus entre elles, le "script" gardera invariablement son sens. A lire Barthes de cet oil cognitif, on est frappé par la similitude entre la notion de "script " et celle du code proaïrétique qu'il présente dans $S / Z(1970$, p. 26) :
[...] la séquence proaïrétique n'est jamais que l'effet d'un artifice de lecture: quiconque lit le texte rassemble certaines informations sous quelque nom générique d'actions (Promenade, Assassinat, Rendez-vous), et c'est le nom qui fait la séquence.

Ainsi, une exploitation aussi radicale du "script» au cinéma se traduira par la présentation d'une seule et courte "scène de script" et la suppression des autres formant l'événement typique. C'est le cas entre autres de ces enlacements passionnés que l'on retrouve dans de nombreuses comédies sentimentales, enlacements qui se terminent sur un plan des jambes du couple alors que l'on voit tomber la robe aux pieds du personnage féminin. Ce seul plan permet de suggérer le «script étreinte amoureuse" qui va suivre. C'est le fameux "principe des etc." de Gombrich (1989, p. 220): «[...] the assumptions we tend to make that to see few members of a series is to see them all ».

En fait, le cinéma est un art de l'ellipse qui, suivant la démonstration qui a précédé, ressemble davantage au " $\mathrm{C}^{*} \mathrm{NE}^{*} \mathrm{AT} \mathrm{T}^{*} \mathrm{GRA}{ }^{*} \mathrm{HE}$ " qu’à une représentation intégrale. La durée définie et immuable de la projection entraîne plusieurs choix, tant sur le plan de la mise en film que sur celui de la mise en intrigue. Le cadrage et le montage constituent les déterminations les plus évidentes, déterminations qui n'entraînent certes pas toujours des hiatus insignifiants. Mais qu'importe puisque, écrit Paul Valéry, c'est le manque et la lacune qui créent. Philippe Durand (1993, p. 15), qui cite Valéry dans Cinéma et montage. Un art de l'ellipse, poursuit: "Éclairant aphorisme. Dont le cinéma sait si bien tirer parti. " "Et que la spectature prolonge», ajouterai-je. Car si, comme l'enseigne la narratologie, le récit en dit toujours moins qu'il n'en sait, le spectateur en sait certainement plus que ce que dit le récit. Et on pourrait ajouter qu’à travers les médiateurs 
culturels (bandes-annonces, affiches promotionnelles, synopsis et critiques publiées dans les revues et journaux), les consignes institutionnelles et les horizons d'attente, la spectature va bien souvent au-delà de l'information qui lui est donnée avant même que le récit n’ait « dit un seul mot».

\section{«Aller au-delà » du « recueillir plus»}

Un retour à la conceptualisation de Neisser s'impose. Sous l'angle de la théorie du schéma et de son concept de cycle perceptif - que j'ai pour mon compte transformé en cercle heuristique -, Neisser analyse la compréhension et, surtout, critique la thèse de Bruner (1976, p. 182 - c'est moi qui souligne):

Perceptual cycles vary in the kind and scope of the informations that guide them. Unsophisticated perceivers are tuned to relatively superficial features of the environment; skilled perceivers to subtler ones. The adult is more future oriented and more effectively goal directed than the child, but not more independent of the world around him. Perceiving is a matter of picking up information, not of going beyond it.

Neisser se sert du jeu d'échecs pour illustrer son propos. Il observe que devant un échiquier l'enfant aperçoit des objets à se mettre dans la bouche, le non-joueur y voit la forme des pièces alors que le maitre, lui, perçoit la structure des positions et tous les mouvements possibles. Dès lors, en percevant l'information qui est donnée, on ne cherche pas à "aller au-delà ", mais plutôt à trouver une représentation cognitive d'accueil pour dégager toutes les conclusions non démonstratives nécessaires. C'est en sélectionnant et en recueillant le plus grand nombre d'informations pertinentes que l'on peut relier celles-ci à un schéma de connaissance. On ne va pas au-delà du «C $\mathrm{C}^{*} \mathrm{NE}^{*} \mathrm{AT}^{*} \mathrm{GRA} \mathrm{H}^{*} \mathrm{HE}$ », on le met en relation avec le schéma "CINÉMATOGRAPHE". On peut dire la même chose de la séquence ordinaire au restaurant ou de la réapparition d'une même série de plans qui renvoie au montage alterné. De même, comme pour les échecs, alors que le spectateur novice fera abstraction d'un faux raccord dans sa perception du montage d'une scène, le chercheur expert, lui, 
remarquera particulièrement sa présence puisqu'il en a une connaissance spécifique.

N'empêche que, malgré la différence de terminologie et d'approche, Bruner et Neisser se rejoignent sur la nature de l'activité déployée par un spectateur aussi bien dans un contexte quotidien que dans celui du visionnement d'un film. Je crois même qu'ils ont, le second évidemment plus que le premier, négligé de considérer toute la portée du processus cyclique de la perception et de la cognition. J'avance donc, pour définir la spectature, une expression qui me semble encore plus appropriée que le «aller au-delà de l'information donnée» ou le "recueillir plus d'informations pertinentes». Cette expression, c'est: «faire le tour de la question ». La réflexion sur le cercle heuristique permet en effet de constater que le spectateur doit d'abord examiner les informations qui lui sont fournies pour ensuite déduire les conclusions qui conviennent à la situation. Comme on le sait, il est souvent nécessaire de passer régulièrement en revue les données d'un problème pour réviser ses réactions et ses comportements. Et cela est davantage marqué devant un défilement diachronique. Il est alors nécessaire de continuellement réviser ses hypothèses et ses conclusions. Il faudra ainsi attendre le "T» pour être à même de savoir que le "C $\mathrm{C}^{*} \mathrm{NE}^{*} \mathrm{AT}$... » ne renvoie pas simplement à "CINÉMA». Comme on ne pourra rien conclure avec le « $\mathrm{E}$ " final, ce dernier seul pouvant indiquer que " $\mathrm{C}^{*} \mathrm{NE}^{*} \mathrm{AT}^{*} \mathrm{GRA}{ }^{*} \mathrm{HE}$ » ne renvoie pas à "CINÉMATOGRAPHIE " ni à "CINÉMATOGRAPHIQUE». Au cinéma, il faudra attendre la fin d'un mouvement de caméra, celle d'une scène ou d'un segment pour en saisir toute la portée. Par exemple, au début de Goodfellas (Martin Scorsese, 1990), la caméra commence sa course sur une table, montre le jeune Henry (Christopher Serrone) en train de faire un sandwich et le suit dans une foule de "wiseguys". Il faut attendre la toute fin du plan pour saisir vers quoi ou vers qui nous menait ce mouvement. Ce n'est ni vers l'homme qui a commandé le sandwich ni vers Paulie, le patron (Paul Cicero) qui trône au milieu de la pièce, mais bien vers James "Jimmy" Conway (Robert De Niro) qui fait son entrée.

En définitive, puisqu'il est ici question d'un processus, le spectateur "fait des tours de la question" durant sa progression. 
Qui dit intrigue dit aussi interrogation(s), de préférence au pluriel. Dès lors, le mouvement du récit filmique adhère à un modèle de questions/réponses. Bien que cette relation logique ne soit pas unique au cinéma, elle représente pour Noël Carroll la structure centrale des "movies", à savoir des films de fiction narratifs populaires ${ }^{16}$. Carroll (1988, p. 173) qualifie cette structure de "erotetic narration ", expression désignant la recherche - l'éros — du sens logique de l'action - le thétique. Si la résolution d'une intrigue représente LA question du cinéma narratif, il n'en demeure pas moins qu'au cours d'une spectatureen-progression, il aura fallu enchaîner plus d'un jugement et plus d'une inférence pour aboutir au dénouement final. D'ailleurs, ne fait-on pas attendre le spectateur quatre-vingt-dix minutes avant de lui donner LA clé de l'énigme? En apportant une légère modification à une expression fort connue et fort citée de Barthes (1977, p. 15), on peut dire que l'enjeu sémantique et narratif n'est pas "au bout" du récit filmique, mais qu'il le traverse. C'est dans cette perspective que la narration érothétique de Carroll se comprend le mieux et que, finalement, elle peut se comparer à la résolution de problèmes. Ainsi, chacune des scènes appelle, à divers degrés, une question ou une réponse qui énonce les possibilités narratives ${ }^{17}$. Ces micro-questions/réponses servent à accélérer ou à freiner la progression du récit qui mènera à la résolution de l'intrigue, la macro-réponse du film. Par exemple, le spectateur saura d'abord durant le visionnement d'Exotica (Atom Egoyan, 1994) qu'Éric (Elias Koteas) et Christina (Mia Kirshner) ont été amoureux; ensuite qu'ils se sont rencontrés le jour où Éric trouva assassinée, dans le champ jaune, la petite fille de Francis (Bruce Greenwood), le barbu qui vient voir danser Christina; et qu'enfin, Christina gardait cette petite fille et entretenait une relation de confiance avec Francis, qui représentait pour elle une figure paternelle et protectrice. C'est seulement à la toute fin du film qu'il sera possible de comprendre les étranges relations qu'entretenaient tous les personnages. La narration érothétique fait du spectateur - qu'il en soit conscient ou non ${ }^{18}$ - un "question-former". Cependant, dans la mesure où, selon Carroll (1988, p. 173), ce "producteur de questions" anticipe les réponses tout en s'attendant à ce que 
celles-ci soient données par la narration, l'expression porte à confusion et n'explique pas la nature profonde de la spectature. Puisqu'il lui faut trouver les réponses les mieux adaptées aux questions toujours renouvelées de ce vaste jeu de rébus qu'est un film de fiction narratif, il apparaît de nouveau plus juste d'affirmer que le spectateur doit «faire le tour de la question». Ses incessantes questions/réponses ont des répercussions sur la suite du récit, sur l'éventail des choix qu'il fera subséquemment ainsi que sur l'horizon futur des possibilités. Comme je l'ai noté, il "tourne» autour des micro-questions soulevées par l'intrigue. Lorsqu'il trouve des réponses et que les choses se précisent, il peut abandonner certaines hypothèses qu'il avait formulées. À la fin, il aura fait "un tour" fonctionnel de la ou des macroquestions du film ${ }^{19}$. En revanche, cherchant une cohésion globale et visant l'épuisement des données audiovisuelles et narratives, le chercheur en études cinématographiques refait quant à lui "plusieurs tours » d'un même film. S'il ne s'intéresse qu'aux sens référentiels et littéraux, il gravite toujours dans l'orbite du cercle heuristique. S'il déplace son propos vers les sens symboliques et symptomatiques et la production d'un discours sur le film, il se déplace alors vers le cercle herméneutique. La relation entre ces deux analyses posées demande évidemment à être explicitée. Mettant l'accent sur l'élargissement des connaissances, la figure de la spirale me semble, à ce stade-ci, un bon point de départ. Mais d'entrée de jeu, à l'instar de Gervais qui souligne de manière fort juste qu'on ne peut pas opposer unilatéralement la progression et la compréhension ${ }^{20}$, il est fondamental de noter qu'on ne peut pas plus opposer les activités de compréhension et d'interprétation. Ainsi, avant de rétrograder pour approfondir sa compréhension, le chercheur en études cinématographiques doit obligatoirement avoir progressé en essayant de comprendre, car son interprétation en dépend. Il en est manifestement ainsi lorsque, par plaisir et pour la première fois, il visionne un film qu'il n'analysera pas. Et c'est en effet pour se divertir que le spectateur va habituellement au cinéma. D'autre part, bien qu'il n'ait pas à produire un discours en sortant de la salle de cinéma ou à en faire une analyse, le spectateur peut lui aussi saisir la nature symbolique et réflexive d'un film. Nul besoin d'être 
herméneute pour saisir le sens moral qui se cache derrière The Family Man (Brett Ratner, 2000), ce film qui raconte comment un homme d'affaires prospère et célibataire (Nicolas Cage), plongé par magie dans la petite vie de famille qui aurait été la sienne s'il avait marié sa petite amie Kate (Tea Leoni) treize ans plus tôt, finit par aspirer à une existence moins centrée sur la carrière, le pouvoir et l'argent.

\section{Conclusion}

Les objections que formule Neisser à la théorie de Bruner ont pour contexte le débat entourant la manipulation et le contrôle du comportement humain ou, dans le présent cadre d'étude, de l'activité perceptivo-cognitive du spectateur. Pour Neisser, le "aller au-delà de l'information donnée" implique un contrôle trop direct du spectateur. Contrôle grâce auquel l'adulte aurait sur l'enfant l'avantage d'une compétence supérieure et celui de dominer son environnement. Ainsi, l'expression de Bruner peut laisser croire que l'" $[\ldots]$ attention "changes from being captured to being exploratory" [...]» (Neisser, 1976, p. 17921), proposition qui résiste difficilement à un raisonnement plus approfondi.

No choice is ever free of the information on which it is based. Nevertheless, the information is selected by the chooser himself. On the other hand, no choice is ever determined by the environment directly. Still, that environnement supplies the information that the chooser will use (Neisser, 1976, p. 182).

On retrouve ici le paradoxe de la partie et du tout, de la perception et de l'anticipation. En ce sens, la conceptualisation du cercle heuristique brise ce que l'étude de la vision romanesque et l'analyse $\mathrm{du}$ récit cinématographique théorisent sous le nom de point de vue ou d' " œil-caméra" (Jost) et qui leur permet de "[...] traiter la description comme la vision attestée, le récit comme le témoignage oculaire, le discours comme le réel, et la diégèse comme préexistant au récit» (Vernet, 1990, p. 30). Ce n'est pas la représentation qui pivote autour d'un sujet - le point de la vue, d'où l'on dessinerait la pyramide visuelle imagi- 
naire d'un champ oculaire -, mais bien l'activité perceptivocognitive du spectateur qui «fait le tour de la question ", c'est-àdire du film et de l'intrigue. On le sait, il n'existe pas de centre fixe qui reçoive passivement des stimuli. Comme le note Neisser (1976, p. 11), les expériences passées ont des conséquences sur celles qu'on vit et qu'on perçoit:

Perception and cognition are usually not just operations in the head, but transactions with the world. These transactions do not merely inform the perceiver, they also transform him. Each of us is created by the cognition acts in which he engages.

Le film ne préexiste pas à la perception; il est plutôt le résultat d'une création cognitive.

In summary, when a film is experienced fictionnaly, reference is not to the profilmic event in which a set is decorated and actor given direction, but rather to a postfilmic event in which patterns are discovered through active perceiving that affects the overall structure of our knowledge (Branigan, 1992, p. 200).

Sur le plan cognitif, la notion d'interaction doit donc être préférée à celle de communication, pour comprendre la spectatureen-progression. Bien que la narration oriente l'activité perceptivo-cognitive du spectateur, ce dernier traite continuellement l'information qui lui est présentée. Il actualise la signification à partir de son savoir préalable, il est continuellement obligé de réviser sa représentation mentale globale en cours de progression et impose finalement ses propres cadres d'expérience au film. C'est dans l'interaction du mode de perception ascendant ("de bas en haut») et du mode de traitement descendant ("de haut en bas") que le spectateur perçoit et comprend. Ce sont les jeux et les enjeux de cette interaction qui doivent notamment retenir l'attention des futures recherches de l'approche cognitive du cinéma.

Université de Montréal

"Faire le tour de la question» 


\section{NOTES}

1. Cet article a été rédigé avec le support du Conseil de recherches en sciences humaines du Canada. Je tiens à remercier l'un des évaluateurs externes pour son évaluation plus que rigoureuse. Ses commentaires nombreux et constructifs m'ont permis d'affiner mon propos et d'approfondir ma réflexion.

2. Selon Hochberg et Brooks, la perception en direct caractérise le premier visonnement d'un film (qui sera souvent le seul que fera le spectateur), l'analyse posée (ou "leisurely analysis") caractérisant quant à elle les analyses effectuées en dehors de la continuité temporelle du film et qui s'intéressent à ce qui doit être compris d'un film, par exemple les structures des récits, les niveaux de sens, etc. Bertrand Gervais (1993) opère une distinction similaire en études littéraires. Il définit deux régies de lecture: la lecture-en-compréhension et la lecture-en-progression. La lecture-en-compréhension, ou lecture littéraire intensive, cherche à comprendre le texte en profondeur et à en déployer les significations. Cette régie est ultimement productrice d'un discours susceptible d'être évalué et jugé par l'institution littéraire ou par des communautés interprétatives, dont le rôle est d'imposer des formes discursives, des interprétations, des valeurs qui servent à déterminer la justesse et la fausseté des discours tenus sur les textes (p. 115). La lecture-en-progression, ou compréhension fonctionnelle, s'attache à effectuer une première saisie du texte. Le but explicite n'est pas tant de tout comprendre que de progresser plus avant, de prendre connaissance du texte. La mise en intrigue amène souvent le lecteur à vouloir rechercher la suite du récit. Il y a, d'une certaine façon, "suspense", une attente qui pousse à aller de l'avant, au détriment d'une plus grande précision dans notre compréhension des événements (p. 46). Puisque le spectateur n'a pas le choix de progresser dans le récit filmique, d'en suivre le rythme et de le franchir d'un trait à cause de l'irréversibilité temporelle, la lecture-enprogression est encore plus fondamentale au cinéma. Pour cette raison, on parlera d'emblée de spectature-en-progression.

3. C'est pourquoi je le nomme "mode de traitement", le distinguant ainsi du mode de processus ascendant qui, lui, repose spécifiquement sur les mécanismes de la perception.

4. Cette métaphore rend compte de "[...] l'enchaînement et de l'articulation des sous-processus de traitement du message. Elle pose les données perceptives "en bas", en "entrée" ou en "périphérie" du système, alors que le contenu significatif, ou encore la représentation sous forme de "concepts", se trouve "en haut" et est "centrale" "(Weil-Barais, 1993, p. 224).

5. À l'exception de Joseph Anderson (1996), aucun chercheur ne renvoie, à ma connaissance, aux travaux de Neisser. Avant d'être cité par Joseph et Barbara Anderson (1996) et ainsi faire partie de la bibliographie sélective de Post-Theory (Bordweli et Carroll, 1996), Cognition and Reality ne se trouvait pas dans les références introductives de "A Case for Cognitivism" de Bordwell (1989b) ni dans la bibliographie de Narrative Comprehension and Film de Branigan (1992).

6. L'herméneutique ne se réduit évidemment pas à cette définition. On se reportera notamment à "Six Modern Definitions of Hermeneutics" de Richard E. Palmer (1969, p. 33-45). Par ailleurs, un survol rapide de la pensée herméneutique énonce remarquablement bien l'obstacle premier qui se dresse devant toute application authentique de cette pratique au cinéma. On le sait, l'herméneutique dérive de l'exégèse biblique et de l'interprétation des Saintes Écritures. Par la suite, l'écrit semble voué au sacrement. Dans "Origine et développement de l'herméneutique (1900)", Dilthey définit cette science de l'esprit comme "l'art d'interpréter des monuments écrits" (1947, p. 322), ou comme "[...] cette technique de l'interprétation des manifestations vitales fixées par écrit" (p. 334). C'est précisément parce que les monuments écrits peuvent fixer des témoignages humains de façon durable qu'ils sont sus- 
ceptibles de posséder une validité générale. Cette condition fondamentale chez Dilthey est réitérée chez Gadamer (1976, p. 242) et Ricœur (1986, p. 76). Le premier déclare que «tout écrit est par excellence objet d'herméneutique». Le second, quant à lui, note que «[1] a première localité que l'herméneutique entreprend de désenclaver, c'est assurément le angage et plus particulièrement le langage écrit ». Partant, Gadamer et Ricœur relégitiment la place accordée par Dilthey aux monuments écrits, place que Schleiermacher avait auparavant mise en doute. En 1829, Schleiermacher avait élargi le domaine de l'herméneutique: "[...] la solution de la tâche, pour laquelle nous cherchons justement la théorie, n'est nullement liée à l'état du discours que l'écriture peut fixer pour l'œil, mais se trouve chaque fois qu'il s'agit de percevoir, par l'intermédiaire des mots, des idées ou des séries d'idées" (1987, p. 162, c'est moi qui souligne). Mais bien que nous puissions voir là une plus grande ouverture de l'art de l'interprétation, l'acte de compréhension demeure tout de même attaché à une théorie du langage. En s'appuyant sur l'esprit de l'Antiquité qui se reconnaît également dans les arts plastiques, Schleiermacher (1987, p. 184) prend garde de préciser en notes marginales que l'herméneutique "[...] ne peut avoir affaire qu'à ce qui est produit dans la langue". L'écrit demeure hiératique.

7. Le "script» est un schéma ou une représentation cognitive définie et typique d'événements, d'actions et de conduites sociales (le "script" RESTAURANT, par exemple), alors que la "scène" est un schéma ou une représentation cognitive définie et typique de relations spatiales ou physiques d'objets dans un lieu commun (par exemple, la "scène" SALLE DE CINÉMA).

8. Je modifie ici quelque peu la figure introduite dans mon article «Une machine à faire penser" (Perron, 1995, p. 79). Cette seconde version est, comme l'essentiel de la présente réflexion, tirée du chapitre cinq de ma thèse de doctorat (Perron, 1997, p. 107-135).

9. Un film entretient des relations d'échos, de résonances, de contrastes, de similitudes ou de réitérations avec d'autres films. Ce "vaste champ d'interférences" (Gardies et Bessalel, 1992, p. 125) ou ce grand espace discursif est habituellement désigné sous le nom d'intertextualité. Je dirai plutôt que, pour être accessible et compréhensible, tout film requiert un "co-texte" (une expression empruntée à Eco, 1985 p. 19), de même que toute interprétation nécessite un contexte, c'est-à-dire "[...] une construction psychologique, un sous-ensemble des hypothèses de l'auditeur sur le monde» (Sperber et Wilson, 1989, p. 31).

10. En ce sens, les tons de gris de la figure correspondent à ceux des deux derniers niveaux de l'architecture mémorielle que j'ai présentés ailleurs (Perron, 1994, p. 93). Le ton plus gras de l'intérieur du cercle renvoie à la mémoire de travail et le ton plus pâle de l'extérieur, à la mémoire à long terme.

11. Plus particulièrement, il s'agit d'un article de Jerome Bruner publié pour la première fois en 1957.

12. Il s'agissait de lire à voix haute deux histoires ("Truth and Consequence" de Brenda Gill et "Nostalgia : or Grease Spots" de Henrich Böll pour la première étude; "Eveline" de James Joyce et "A Respectable Woman" de Kate Chopin pour la seconde) selon deux versions différentes (l'une tenant compte de la conscience du protagoniste et l'autre, non) et, suivant quelques variations, de poser des questions au sujet en cours de lecture et à la fin celle-ci. Par exemple, au quart de l'histoire, on demandait au sujet: "Quelle est la chose la plus importante que je vous ai dite jusqu’à maintenant? Pourquoi?" Aux trois quarts de l'histoire, on demandait: "Dans quelles directions cela peut aller? Dans quelle direction pensez-vous que cela va aller? Pourquoi ? " Etc.

13. Bruner et ses confrères opposent à ce paysage le paysage de la conscience ("landscape of consciousness") qui renvoie non pas à une activité de compréhension 
("explanation") mais à une activité d'interprétation et même d'exégèse (Feldman, Bruner, Rendered et Spitzer, 1990, p. 3). Ce second paysage serait donc davantage associé au cercle herméneutique. Seulement, comme je le noterai plus loin, on ne peut pas considérer que la compréhension et l'interprétation soient en complète opposition.

14. Bruner note qu'avant même l'acquisition du langage et la maîtrise des propositions logiques les plus fondamentales, l'enfant produit et comprend des histoires. Il existe une «impulsion" vers la construction de récits qui détermine l'ordre de priorité dans lequel les formes grammaticales sont maîtrisées. En ce sens, les "unités naturelles» de la communication ne sont pas les phrases mais les unités de discours, ces dernières remplissant une fonction pragmatique (faire agir les autres pour son compte) ou une fonction mathétique (rendre claires ses pensées sur le monde) (Bruner, 1991, p. 88).

15. Par le terme "scène" de script" (noter les guillemets qui distinguent ce concept du précédent), Jean Matter Mandler (1984, p. 77) désigne des "action[s] organized around the subgoals involved in achieving the main goal of the script». Il s'agira par exemple des actions énumérées dans le "script " RESTAURANT.

16. Carroll distingue les "movies" - paysage de l'action (Feldman et al., 1990), récits populaires (Rabinowitz) ou textes lisibles (Barthes) - des "films" (paysage de la conscience, récits sérieux ou textes scriptibles). Alors que les "movies" sont fonctionnels par leur respect de l'ordre du monde et de la logique causale, qu'ils sont conditionnés par l'intrigue et insistent sur des opérations de configuration dans la mesure où l'on demande au spectateur de regrouper des traits caractéristiques en modèles ou schémas connus, les «films" de leur côté sont davantage indiciels, plus ambigus parce que reposant sur l'imperfection du monde et la confusion de la vie, ils sont conditionnés par les personnages et ont tendance à renforcer les opérations de signification et de cohérence, à savoir que le spectateur est amené à chercher le sens et la cohésion d'un élément du film ou des réactions d'un personnage. Bien que Carroll (1988, p. 176) considère que son modèle de questions/réponses ne s'applique pas aux "films", il fonctionne certainement sur un autre plan. Certes, si " [m] odernists exercices like Last Year in Marienbad and India Song literally defy the erotetic model they are all questions with no answers ", ils n'exemptent pas pour autant le spectateur de "faire le tour des questions" posées, questions qui ne sont de toute évidence pas de type causal. Les "answering scenes " étant moins nombreuses et souvent inexistantes, une question en attire une autre. Le spectateur n'est pas tant amené à répondre à des questions qu'à s'en poser davantage — à propos du "film", du cinéma et du monde en général - et à trouver ses propres réponses.

17. Carroll (1988, p. 174-175), s'inspirant des théories de Poudovkine (Film Technique and Film Acting), identifie sept catégories de scènes: 1) an esthablishing scene;2) a questioning scene; 3) an answering scene; 4) a sustaining scene; 5) an incomplete answering scene ; 6) an answering/questioning scene; et 7) a fulfilling scene.

18. Carroll (1988, p. 173) mentionne qu'il ne faut pas confondre les processus mentaux. Ainsi, "avoir une question en tête" n'équivaut pas nécessairement à «se livrer à un questionnement mental conscient". Le spectateur a des attentes subconscientes. Par exemple, si l'on arrête la projection d'un film à mi-chemin, LA question au sujet du dénouement fait très vite surface.

19. Ce tour suffira au spectateur qui va au cinéma pour se divertir, dans la mesure où la narration érothétique lui aura facilité la tâche: "A successful erotetic narrative tells you, literally, everything you want to know about the action being depicted, i.c., it answers every question, or virtually every question that it has chosen to pose saliently. [...] Unlike those of real life, the actions observed in movies has level of intelligibility, due to the role they play in the erotetic system of questions and ans- 
wers. Because of the question/answer structure, the audience is left with the impression that it has learned everything important to know concerning the action depicted" $(1988$, p. 180).

20. "Lire, c'est progresser et comprendre, et l'importance accordée à l'une ou l'autre de ces économies dépend des objectifs du lecteur, de ses mandats. Les différences de lecture (ou de mandats de lecture) sont donc fonction de la prépondérance de l'une ou l'autre de ces économies: comprendre mieux ou progresser plus en avant" (Gervais, 1993, p. 43).

21. Les guillemets dans la citation sont de Bruner, ce dernier citant Eleanor J. Gibson qui va dans la mêrne direction que lui.

\section{RÉFÉRENCES BIBLIOGRAPHIQUES}

Anderson, 1996: Joseph Anderson, The Reality of Illusion: An Ecological Approach to Film Cognitive Theory, Carbondale et Edwardsville, Southern Illinois University Press, 1996.

Anderson et Anderson, 1996 : Joseph et Barbara Anderson, «The Case for an Ecological Metatheory", dans David Bordwell et Noël Carroll (dir.), Post-Theory: Reconstructing Film Studies, Madison, The University of Wisconsin Press, 1996, p. 347-367.

Barthes, 1970 : Roland Barthes, S/Z, Paris, Seuil, 1970.

Barthes, 1977 : Roland Barthes, "Introduction à l'analyse structurale des récits ", Poétique du récit, Paris, Seuil, [1966] 1977, p. 7-57.

Bordwell, 1985: David Bordwell, Narration in the Fiction Film, Madison, University of Wisconsin Press, 1985.

Bordwell, 1989a : David Bordwell, "A Case for Cognitivism ", Iris, n 9, 1989, p. 1140.

Bordwell, 1989b: David Bordwell, Making Meaning: Inference and Rhetoric in the Interpretation of Cinema, Cambridge, Harvard University Press, 1989.

Bordwell et Carroll, 1996: David Bordwell et Noël Carroll (dir.), Post-Theory: Reconstructing Film Studies, Madison, The University of Wisconsin Press, 1996.

Branigan, 1992: Edward Branigan, Narrative Comprehension and Film, New York, Routledge, 1992.

Bruner, 1957 : Jerome S. Bruner, "Going Beyond the Information Given», dans H. E. Gruber, K. R. Hammond, et R. Jessor (dir.), Contemporary Approaches to Cognition, Cambridge, Harvard University Press, 1957, p. 41-69.

Bruner, 1973 : Jerome S. Bruner, Beyond the Information Given: Studies in the Psychology of Knowing, New York, W.W. Norton \& Company, 1973.

Bruner, 1991 : Jerome S. Bruner, Car la culture donne forme à l'esprit. De la révolution cognitive à la psychologie culturelle, Paris, Éditions Eshel, [1990] 1991.

Carroll, 1988: Noël Carroll, Mystifying Movies: Fads \& Fallacies in Contemporary Film Theory, New York, Columbia University Press, 1988.

Dilthey, 1947: Wilhelm Dilthey, Le Monde de l'esprit, Paris, Aubier-Montaigne, 1947.

Durand, 1993: Philippe Durand, Cinéma et montage. Un art de l'ellipse, Paris, Éditions du Cerf, 1993.

Eco, 1985: Umberto Eco, Lector in fabula. Le rôle du lecteur, Paris, Éditions Grasset et Fasquelle, [1979] 1985.

Eco, 1994 : Umberto Eco, Six Walks in the Fictional Woods, Cambridge, Harvard University Press, 1994. 
Ellis et Hunt, 1989 : Henry C. Ellis et R. Reed Hunt, Fundamentals of Human Memory and Cognition, Iowa, W. C. Brown Publishers.

Fayol, 1985: Michel Fayol, Le Récit et sa construction. Une approche de psychologie cognitive, Neuchâtel et Paris, Delachaux et Niestlé, 1985.

Feldman et al., 1990 : Carole Fleisher Feldman, Jerome S. Bruner, Bobbi Rendered et Sally Spitzer, "Narrative Comprehension", dans Bruce K. Briton et A.D. Pellegrin (dir.), Narrative Thought and Narrative Language, New Jersey, Lawrence Erlbaum Associates, 1990, p. 1-78.

Fortin et Rousseau, 1993 : Claudette Fortin et Robert Rousseau, Psychologie cognitive. Une approche du traitement de l'information, Québec, Télé-Université, 1993.

Gadamer, 1976: Hans-Georg Gradamer, Vérité et méthode. Les grandes lignes d'une herméneutique philosophique, Paris, Éditions du Seuil, [1960] 1976.

Gardies et Bessalel, 1992: André Gardies et Jean Bessalel, 200 mots-clés de la théorie du cinéma, Paris, Éditions du Cerf, 1992.

Gardies, 1993: André Gardies, L'Espace au cinéma, Paris, Méridiens Klincksieck, 1993.

Gervais, 1993: Bertrand Gervais, À l'écoute de la lecture, Montréal, VLB Éditeur, 1993.

Goffman, 1991 : Erving Goffman, Les Cadres de l'expérience, Paris, Éditions de Minuit, [1974] 1991.

Hochberg et Brooks, 1996: Julian Hochberg et Virginia Brooks, "Movies in the Mind's Eye", dans David Bordwell et Noël Carroll (dir.), Post-Theory: Reconstructing Film Studies, Madison, The University of Wisconsin Press, 1996, p. 368-387.

Mandler, 1984: Jean Matter Mandler, Stories, Scripts, and Scenes: Aspects of Schema Theory, Hillsdale, Lawrence Erlbaum Associates, 1984.

Metz, 1968 : Christian Metz, Essais sur la signification au cinéma, tome 1, Paris, Klincksieck, 1968.

Neisser, 1976: Ulric Neisser, Cognition and Reality: Principles and Implications of Cognitive Psychology, San Francisco, W.H. Freeman and Company, 1976.

Palmer, 1969: Richard E. Palmer, Hermeneutics: Interpretation Theory in Schleiermacher, Dilthey, Heidegger and Gadamer, Evaston, Northwestern University Press, 1969.

Perron, 1994: Bernard Perron, "La mémoire, c'est ce qu'il me reste à défaut d'une vue », Cinémas, vol. 5, nos 1-2, 1994, p. 91-103.

Perron, 1995: Bernard Perron, "Une machine à faire penser", Iris, n 20, 1995, p. 76-84.

Perron, 1997 : Bernard Perron, "La Spectature prise au jeu. La narration, la cognition et le jeu dans le cinéma narratif", thèse de doctorat, Université de Montréal, 1997.

Rabinowitz, 1987 : Peter J. Rabinowitz, Before Reading: Narrative Conventions and the Politics of Interpretation, Ithaca, Cornell University Press, 1987.

Ricceur, 1986: Paul Ricour, Du texte à l'action. Essais d'herméneutique II, Paris, Éditions du Seuil, 1986.

Schleiermacher, 1987: Fredrich Daniel Ernst Schleiermacher, Herméneutique. Pour une logique du discours individuel, Paris, Éditions du Cerf et Presses de l'Université de Lyon, 1987.

Sperber et Wilson, 1989 : Dan Sperber et Deirde Wilson, La Pertinence. Communication et Cognition, Paris, Éditions de Minuit, [1986] 1989.

Thérien, 1992: Gilles Thérien, "La lisibilité au cinéma», Cinémas, vol. 2, nos 2-3, 1992, p. 107-122. 
Vernet, 1990 : Marc Vernet, «D'un oil peu regardant ", La Licorne, $\mathrm{n}^{\circ}$ 17, Université de Poitiers, 1990, p. 29-38.

Weil-Barais, 1993 : Annick Weil-Barais (dir.), L'Homme cognitif, Paris, Presses universitaires de France, 1993. 